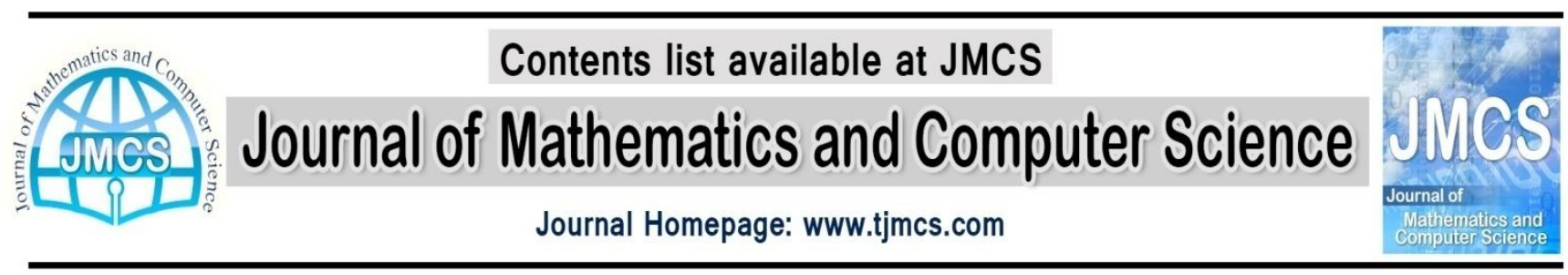

\title{
A generalization of iteration-free search vectors of ABS methods
}

\author{
Mostafa Khorramizadeh \\ Department of Mathematical Sciences \\ Shiraz University of Technology \\ Shiraz 71555-313, Iran
}

Article history:

Received July 2014

Accepted August 2014

Available online August 2014

\section{m.khorrami@sutech.ac.ir}

\begin{abstract}
Recently, we introduced iteration-free search vectors of the ABS methods and showed how they can be used to compute the search directions of primal--dual interior point methods, when the coefficient matrix of the constraints of the linear programming problem is square. Here, we generalize those results for the general case when, the coefficient matrix is non-square.
\end{abstract}

Keywords: Interior point methods, Infeasible interior pointmethods, Primal--dual algorithms, ABS algorithms, Searchdirection.

\section{Introduction}

Assume that $A$ is an $m \times n$ matrix with $\operatorname{rank}(A)=m$. Let $c, x$ and $s$ be $n$-vectors and $b$ be an $m$-vector. Then, the primal linear programming problem $[5,7]$ is defined to be the minimization of the objective function $c^{T} x$ subject to the functional constraints $A x=b$ and the non-negativity constraints $x \geq 0$. The dual of this problem is then the maximization of $b^{T} y$ subject to $A^{T} y+s=c$ and $s \leq 0$. In the $k$ th iteration of primal--dual infeasibleinterior point algorithms the search direction is computed by solving the following system of linear equations $[3,8,9]$ :

$$
\left(\begin{array}{ccc}
0 & A^{T} & I_{n} \\
A & 0 & 0 \\
S^{k} & 0 & X^{k}
\end{array}\right)\left(\begin{array}{c}
\Delta x^{k} \\
\Delta \lambda^{k} \\
\Delta s^{k}
\end{array}\right)=\left(\begin{array}{c}
-r_{c}^{k} \\
-r_{b}^{k} \\
-r_{x s}^{k}
\end{array}\right)
$$

where $r_{c}^{k}, r_{b}^{k}$ and $r_{x s}^{k}$ are given by $r_{b}^{k}=A x^{k}-b, r_{c}^{k}=A^{T} \lambda^{k}+s^{k}-c, r_{x s}^{k}=-X^{k} S^{k} e+\sigma_{k} \mu_{k} \mathbf{1}$. and $X^{k}$ and $S^{k}$ denote the diagonal matrices whose diagonal elements are the components ofthe vectors $x^{k}$ 
and $s^{k}$, respectively, and $\mathbf{1}=(1, \ldots, 1)^{T} \in R^{n}$. Moreover, $\sigma_{k} \in(0,1)$ and $\mu_{k}=\frac{\left(x^{k}\right)^{T} s^{k}}{n}$ are centering parameterand duality gap, respectively.

The ABS class of algorithms was first introduced by Abaffy, Broyden and Spedicato $[1,2,6]$ for solving linear systems. For simplicity in notation, assume $R^{m \times n}$ has full row rank. An ABS method for solving the linear system $A x=b$, with $b \in R^{m}$, starts with anarbitrary initial vector $x_{1} \in R^{n}$ and an arbitrary nonsingular matrix $H_{1} \in R^{n \times n}$, the so-called Spedicato's parameter. In the $i$ th iteration, having computed $x_{i}$, asolution of the first $i-1$ equations of $A x=b$, and $H_{i}$ a matrix with rowsgenerating the null space of the first $i-1$ rows of $A$, an ABS algorithm computes $x_{i+1}$ asa solution of the first $i$ equations of $A x=b$ and $H_{i+1}$, with rows generating the null space of the first $i$ rows of $A$ as explained below [1,2]. To compute the search vector, $z_{i}$ (Broyden's parameter) is determined so that $z_{i}^{T} H_{i} a_{i} \neq 0$ and the search vector is set to be $q_{i}=H_{i}^{T} z_{i}$. Then, the solution is updated by $x_{i+1}=x_{i}+\alpha_{i} q_{i}$, where the step size $\alpha_{i}$ is given by $\alpha_{i}=\left(b_{i}-a_{i}^{T} x_{i}\right) / a_{i}^{T} q_{i}$. Next, $H_{i+1} \$$ is computed so that $H_{i+1} a_{j}=0$, $1 \leq j \leq i$. This can be accomplished by updating $H_{i}$ (the so called Abaffian) by

$$
H_{i+1}=H_{i}-H_{i} a_{i} w_{i}^{T} H_{i} / w_{i}^{T} H_{i} a_{i}
$$

with $w_{1} \in R^{n}$ (Abaffy's parameter) satisfying $w_{i}^{T} H_{i} a_{i} \neq 0$. It can be shown that [1] in an ABS algorithm, we have $s_{i}=H_{i} a_{i} \neq 0 \$$ if and only if $a_{i}$ is linearly independent of $a_{1}, a_{2}, \ldots, a_{i-1}$ (orequivalently, $a_{i}=0$ if and only if $a_{i}$ is linearly dependent on $a_{1}, a_{2}, \ldots, a_{i-1}$. The rows of $H_{i+1}$ generate thenull space of the first $i$ rows of $A$. If $\operatorname{rank}(A)=m$ then every solution of the first $i$ equations of the system can bewritten as $x_{i+1}+H_{i+1}^{T} s$, for some choice of $s \in R^{n}$. Let $x^{*}$ be the special solution of the linear system $A x=b$, then there exist a vector $s^{*} \in R^{n}$ such that $x^{*}=x_{i+1}+H_{i+1}^{T} s^{*}$. Indeed let $r_{i+1}=b-A x_{i+1}$, $Z=\left(H_{i+1} a_{i+1}, \ldots, H_{i+1} a_{m}\right)^{T}$ and $d$ be the solution of the linear system $Z Z^{T} d=\left(r_{i+1}\right)_{m-i}$ where $\left(r_{i+1}\right)_{m-i}$ denotes the last $m-i$ components of the vector $r_{i+1}$. Then, we have $s^{*}=Z^{T} d$. If $x_{1}=0$, then the solution of the system is $P \tau$, where $\mathrm{P}=\left(p_{1}, \ldots, p_{m}\right)$ and $\tau=\left(\tau_{1}, \ldots, \tau_{m}\right)$.

In Section 2, we describe the ideas of iteration-free search vectors of the ABS algorithm for solving (1). In Section 3, we show how we can use these iteration-free search vectors, to characterize the ABS solution of (1), in case $m<n$. Section 4 is devoted to the concluding remarks.

\section{Iteration-free search vectors}

In the $k$ th iteration of primal-dual IIPMs to solve linear optimization problems, the search direction $\left(\Delta x^{k}, \Delta \lambda^{k}, \Delta s^{k}\right)$ is computed by solving the linear system (1). We start the ABS algorithm with $x_{1}=0 \in R^{2 n+m}$ and $H_{1}=I_{2 n+m}$, where $I_{2 n+m}$ is the identity matrix. Then, it can be easily verified that for $1 \leq i \leq n$, if we let $z_{i}=w_{i}=\left(0,0, e_{i}^{T}\right) \in R^{2 n+m}$, where $e_{i}$ is the $i$ th column of the identity matrix $I_{n}$, then, in the $i$ th iteration of the ABS algorithm forsolving (1), we have

$$
p_{i}=\left(\begin{array}{c}
0 \\
0 \\
e_{i}
\end{array}\right), \quad H_{i+1}=\left(\begin{array}{ccc}
I_{n} & 0 & 0 \\
0 & I_{m} & -\sum_{j=1}^{i} \hat{a}_{j} e_{j}^{T} \\
0 & 0 & I_{n}-\sum_{j=1}^{i} e_{j} e_{j}^{T}
\end{array}\right),
$$

where $\hat{a}_{j}$ is the $j$ th column of the matrix $A$. Now let $a_{i}$ 's, $1 \leq i \leq m$, denote the $i$ th row of the matrix $A$. By applying the $A B S$ algorithm to the system $A \Delta x^{k}=-r_{b}^{k}$, starting with $\bar{H}_{1}=I_{n}$ and $\bar{x}_{1}=0 \in R^{n}$, we obtain the parameters $\bar{z}_{i} \in R^{n}, \bar{p}_{i} \in R^{n}$ and $H_{i+1} \in R^{n \times n}$, for $1 \leq i \leq m$ so that $\bar{z}_{i}^{T} \bar{H}_{i} a_{i} \neq 0, \bar{w}_{i}^{T} \bar{H}_{i} a_{i} \neq$ $0, p_{i}=\bar{H}_{i}^{T} \bar{z}_{i}, \bar{H}_{i+1}=\bar{H}_{i}-\bar{H}_{i} a_{i} \bar{w}_{i}^{T} \bar{H}_{i} / \bar{w}_{i}^{T} \bar{H}_{i} a_{i}$. It can be easily verified that for $1 \leq i \leq m$, if we let $z_{n+i}=\left(\bar{z}_{i}, 0,0\right)^{T} \in R^{2 n+m}, w_{n+i}=\left(\bar{w}_{i}, 0,0\right)^{T} \in R^{2 n+m}$, where $\bar{w}_{i}$ and $\bar{z}_{i}$ are defined as above, then, in the $(n+i)$ th iteration of the ABS algorithm applied to solve (1), we have 


$$
p_{n+i}=\left(\begin{array}{c}
\bar{p}_{i} \\
0 \\
0
\end{array}\right), \quad H_{n+i+1}=\left(\begin{array}{ccc}
\bar{H}_{i+1} & 0 & 0 \\
0 & I_{m} & -A \\
0 & 0 & 0
\end{array}\right),
$$

Let $\tilde{A}=\left(\tilde{a}_{1}, \ldots, \tilde{a}_{n}\right)$, where the $i$ th column of this matrix is constructed asfollows. Let $|x|^{2}=\sum_{i=1}^{n} x_{i}^{2}$ and $\breve{a}_{1}=\hat{a}_{1}, \tilde{a}_{1}=$. Now, we define $\breve{a}_{i}=\hat{a}_{i}-\sum_{j=1}^{i-1}\left(\tilde{a}_{j}^{T} \hat{a}_{i}\right) \tilde{a}_{j}$, for $2 \leq i \leq n$ and $\tilde{a}_{i}=\breve{a}_{i} /\left\|\breve{a}_{i}\right\|$, for $2 \leq i \leq n$. The vectors $\tilde{a}_{j}, 1 \leq j \leq m$, are orthonormal. Using $\tilde{A}$, we define the matrices $N_{j} \in R^{n \times m}$, $M_{k}^{j} \in R^{n \times m}, C_{j} \in R^{m \times m}$ and $B_{k}^{j} \in R^{n \times m}$, for $1 \leq j \leq m$, according to the followingrelations:

$$
N_{j}=-\frac{1}{\left\|\check{a}_{i}\right\|} e_{j} e_{j}^{T} \tilde{A}^{T}, M_{k}^{j}=\frac{s_{j}^{k}}{x_{j}^{k}} N_{j}, C_{j}=I_{m}-\sum_{j=1}^{i} \tilde{A} e_{j} e_{j}^{T} \tilde{A}^{T}
$$

and

$$
B_{k}^{1}=H_{m+1} M_{k}^{1}, \quad B_{k}^{j}=B_{k}^{j-1}+B_{k}^{j-1} A N_{j} C_{j-1}-\bar{H}_{m+1} M_{k}^{j} C_{j-1}
$$

In the following Theorem, we provide the ABS parameters forthe $(n+m+i)$ th, $1 \leq i \leq m$, iteration of the algorithmapplied to solve (1). The proof can be found in [4].

Theorem: For $1 \leq i \leq m$, let

$$
w_{n+m+i}=z_{n+m+i}=\left(\begin{array}{c}
0 \\
\tilde{A} e_{i} \\
0
\end{array}\right) \in R^{2 n+m}
$$

Then, in the $(n+m+i)$ th iteration of the ABS algorithmapplied to solve (1), we have

$$
p_{n+m+i}=\left(\begin{array}{c}
0 \\
\tilde{A} e_{i} \\
-A^{T} \tilde{A} e_{i}
\end{array}\right), \quad H_{n+m+i+1}=\left(\begin{array}{ccc}
\bar{H}_{m+1} & B_{k}^{i} & -B_{k}^{i} A \\
0 & C_{i} & -C_{i} A \\
0 & 0 & 0
\end{array}\right),
$$

where the matrices $C_{i}$ and $B_{k}^{i}$ are defined by (5) and(6), respectively.

\section{Computing the search directions}

In this section, we provide the search directions of primal-dual IIPMs using the search vectors obtained in Section 2 for the Newton system (1) for the general case where, $m \leq n$. In this case, we first derive an efficient formula to compute $B_{k}^{m}$. Then, using properties of the ABS algorithm, we characterize the solution of system (1) from the solution of the first $2 n+m$ equations. Consider the case in which $m \leq n$ and $B_{k}^{m}$ is not zero matrix. For $1 \leq i \leq m$, we define

$$
U_{i}^{T}=\left(u_{1}^{i}, \ldots, u_{i}^{i}\right) \in R^{m+i}, \quad D_{i}^{k}=\left(\varepsilon_{1}^{i} e_{1}, \ldots, \varepsilon_{i}^{i} e_{i}\right) \in R^{n+i}
$$

where,

$$
\delta_{j}=\frac{-1}{\left\|\check{a}_{j}\right\|}, \quad \varepsilon_{j}^{k}=-\frac{s_{j}^{k} \delta_{j}}{x_{j}^{k}}, \quad 1 \leq j \leq i
$$

and $u_{1}^{1}=\tilde{A} e_{1}, u_{j}^{i}=u_{j}^{i-1}+\delta_{i} \tilde{A} e_{i} e_{i}^{T} A^{T} u_{j}^{i-1}$, for $1 \leq j \leq i-1$ and $u_{i}^{i}=\tilde{A} e_{i}$. The following theorem provides an efficient formula to compute $B_{k}^{i}, 1 \leq i \leq m$.

Theorem: Let $1 \leq i \leq m$ and the matrices $D_{i}^{k}$ and $U_{i}$ be defined as in (8). Then,

$$
B_{k}^{i}=\bar{H}_{m+1} D_{i}^{k} U_{i}=\bar{H}_{m+1} \sum_{j=1}^{i} \varepsilon_{j}^{k} e_{j}\left(u_{j}^{i}\right)^{T} .
$$

Proof: We proceed by induction. For $i=1$, we have

$$
B_{k}^{1}=\bar{H}_{m+1} D_{1}^{k} U_{1}=\bar{H}_{m+1} \varepsilon_{1}^{k} e_{1}\left(u_{1}^{1}\right)^{T}
$$

which is true by the definition of $B_{k}^{1}$ in (6). Suppose that (10\}) is true up to $i=1,2, \ldots, t-1$. For $i=t$, from (5), (6) and (9) we have

$$
B_{k}^{t}=B_{k}^{t-1}+B_{k}^{t-1} A N_{t} C_{t-1}-\bar{H}_{m+1} M_{k}^{t} C_{t-1}
$$




$$
\begin{gathered}
M_{k}^{t} C_{t-1}=\frac{-s_{t}^{k}}{\left\|\check{a}_{j}\right\| x_{t}^{k}} e_{t} e_{t}^{T} \tilde{A}^{T}\left(I_{m}-\sum_{j=1}^{t-1} \tilde{A} e_{j} e_{j}^{T} \tilde{A}^{T}\right)=-\varepsilon_{t}^{k} e_{t} e_{t}^{T} \tilde{A}^{T} \\
N_{t} C_{t-1}=-\frac{e_{t} e_{t}^{T} \tilde{A}^{T}}{\left\|\check{a}_{t}\right\|}\left(I_{m}-\sum_{j=1}^{t-1} \tilde{A} e_{j} e_{j}^{T} \tilde{A}^{T}\right)=\delta_{t}^{k} e_{t} e_{t}^{T} \tilde{A}^{T}
\end{gathered}
$$

Using the hypothesis of the induction and (11), (12) and (13), we can write

$$
\begin{gathered}
B_{k}^{t}=B_{k}^{t-1}+\delta_{t}^{k} B_{k}^{t-1} A e_{t} e_{t}^{T} \tilde{A}^{T}+\varepsilon_{t}^{k} \bar{H}_{m+1} e_{t} e_{t}^{T} \tilde{A}^{T} \\
=\bar{H}_{m+1} \sum_{j=1}^{t-1} \varepsilon_{j}^{k} e_{j}\left(u_{j}^{t-1}\right)^{T}+\varepsilon_{t}^{k} \bar{H}_{m+1} e_{t} e_{t}^{T} \tilde{A}^{T}+\delta_{t}^{k} \sum_{j=1}^{t-1} \varepsilon_{j}^{k} e_{j}\left(u_{j}^{t-1}\right)^{T} A e_{t} e_{t}^{T} \tilde{A}^{T} \\
=\bar{H}_{m+1}\left(\varepsilon_{t}^{k} e_{t} e_{t}^{T} \tilde{A}^{T}+\sum_{j=1}^{t-1} \varepsilon_{j}^{k} e_{j}\left\{\left(u_{j}^{t-1}\right)^{T}+\delta_{t}^{k}\left(u_{j}^{t-1}\right)^{T} A e_{t} e_{t}^{T} \tilde{A}^{T}\right)\right\} \\
=\bar{H}_{m+1} \sum_{j=1}^{t} \varepsilon_{j}^{k} e_{j}\left(u_{j}^{t}\right)^{T}=\bar{H}_{m+1} D_{t}^{k} U_{t}
\end{gathered}
$$

This completes the induction.

It is worth mentioning that the matrices $U_{i}$ and $\bar{H}_{m+1}$ need to be computed only once in the first iteration of the IIPMs, and $D_{j}^{k}$ is a diagonal matrix. Here, using properties of the ABS algorithm, we construct the solution of system (1) for the case $m \leq n$. Let $S_{(n-m)}^{k}=\sum_{j=m+1}^{n} s_{j}^{k} e_{j} \bar{e}_{j-m}^{T}, X_{(n-m)}^{k}=$ $\sum_{j=m+1}^{n} x_{j}^{k} e_{j} \bar{e}_{j-m}^{T},\left(\bar{Z}^{k}\right)^{T}=\bar{H}_{m+1} S_{(n-m)}^{k}-B_{k}^{t} A X_{(n-m)}^{k}$, where $\bar{e}_{i}$ is the $i$ th column of the identity matrix $I_{n-m}$. Assume that

We note that

$$
\left(Z^{k}\right)^{T}=\left(H_{2 n+m+1}\left(\begin{array}{c}
s_{m+1}^{k} e_{m+1} \\
0 \\
x_{m+1}^{k} e_{m+1}
\end{array}\right), \ldots, H_{2 n+m+1}\left(\begin{array}{c}
s_{n}^{k} e_{n} \\
0 \\
x_{n}^{k} e_{n}
\end{array}\right)\right)=\left(\begin{array}{c}
\left(\bar{Z}^{k}\right)^{T} \\
0 \\
0
\end{array}\right) .
$$

$$
Z^{k}\left(Z^{k}\right)^{T}=\left(\bar{Z}^{k}, 0,0\right)\left(\begin{array}{c}
\left(\bar{Z}^{k}\right)^{T} \\
0 \\
0
\end{array}\right) \bar{Z}^{k}\left(\bar{Z}^{k}\right)^{T}
$$

The residual vector of system (1) in thesolution of the first $2 m+n$ equations is:

$$
r^{k}=\left(\begin{array}{c}
-r_{c}^{k} \\
-r_{b}^{k} \\
-r_{x s}^{k}
\end{array}\right)-\left(\begin{array}{ccc}
0 & A^{T} & I_{n} \\
A & 0 & 0 \\
S^{k} & 0 & X^{k}
\end{array}\right)\left(\begin{array}{c}
P \lambda^{k} \\
\tilde{A} \beta^{k} \\
-r_{c}^{k}-A^{T} \tilde{A} \beta^{k}
\end{array}\right)=\left(\begin{array}{c}
0 \\
0 \\
-r_{x s}^{k}-S^{k} P \lambda^{k}+X^{k} r_{c}^{k}+X^{k} A^{T} \tilde{A} \beta^{k}
\end{array}\right),
$$

where the last equality obtains from the fact that $\left(\left(\lambda^{k}\right)^{T} P^{T},\left(\beta^{k}\right)^{T} \tilde{A}^{T},\left(-r_{c}^{k}-A^{T} \tilde{A} \beta^{k}\right)^{T}\right)$ is the solution of thefirst $2 m+n$ equations. Let $\left(r^{k}\right)_{n-m}$ denotes the last $n-m$ components of the vector $r^{k}$, i.e., $\left(r^{k}\right)_{n-m}=\left(-r_{x s}^{k}-S^{k} P \lambda^{k}+X^{k} r_{c}^{k}+X^{k} A^{T} \tilde{A} \beta^{k}\right)_{n-m}$.Now, let $d^{k}$ satisfies $Z^{k}\left(Z^{k}\right)^{T} d^{k}=\left(r^{k}\right)_{n-m}$. Thus, using properties of the ABS algorithms, the solution of (1) is as follows:

$$
\begin{aligned}
\left(\begin{array}{c}
\Delta x^{k} \\
\Delta \lambda^{k} \\
\Delta s^{k}
\end{array}\right)= & \left(\begin{array}{c}
P \lambda^{k} \\
-\tilde{A} \beta^{k} \\
-r_{c}^{k}-A^{T} \tilde{A} \beta^{k}
\end{array}\right)+\left(\begin{array}{ccc}
\bar{H}_{m+1}^{T} & 0 & 0 \\
\left(B_{k}^{m}\right)^{T} & 0 & 0 \\
-A^{T}\left(B_{k}^{m}\right)^{T} & 0 & 0
\end{array}\right)\left(\begin{array}{c}
\left(\bar{Z}^{k}\right)^{T} d^{k} \\
0 \\
0
\end{array}\right) \\
& =\left(\begin{array}{c}
P \lambda^{k}+\bar{H}_{m+1}^{T}\left(\bar{Z}^{k}\right)^{T} d^{k} \\
-\tilde{A} \beta^{k}+\left(B_{k}^{m}\right)^{T}\left(\bar{Z}^{k}\right)^{T} d^{k} \\
-r_{c}^{k}-A^{T} \tilde{A} \beta^{k}-A^{T}\left(B_{k}^{m}\right)^{T}\left(\bar{Z}^{k}\right)^{T} d^{k}
\end{array}\right)
\end{aligned}
$$




\section{Conclusions}

We generalized iteration free search vectors of the ABS algorithms. Then we used these iteration free search vectors to characterize the solution of the Newton systems of primal-dual infeasible interior point methods.

\section{ACKNOWLEDGEMENTS}

The author thanks Shiraz university of Technology for supporting this work.

\section{References}

[1] J. Abaffy, C. G. Broyden and E. Spedicato, A class of direct methods for linear equations, Numerische Mathematik, 45(1984) 361-376.

[2] J. Abaffy, C. G. Broyden and E. Spedicato, Projection Algorithms: Mathematical Techniques forLinear and Nonlinear Equations, Ellis Horwood, Chichester, 1989.

[3] K. Kojima, N. Megiddo and S. Mizuno, A primal-dual infeasible interior point algorithm for linear programming, Mathematical Programming, 61 (1993), 261-280.

[4] M. Khorramizadeh, On solving Newton systems of Primal-Dual Infeasible Interior Point methods using ABS methods, Applied Mathematical Sciences, 6(106), (2012) 5263-5272.

[5] S. H. Nasseri and Z. Alizadeh,Solving Linear Programming Problem with Fuzzy Right Hand Sides A Penalty Method, The Journal of Mathematics and Computer Science, 3(2011), 318-328.

[6] S. H. Nasseri and F. Zahmatkesh,Huang method for solving fully fuzzy linear system of equations, 1(2005), 1-5.

[7] M. Pattnaik, Fuzzy Muli-Objective Linear Programming Problems Sensivity Analysis, The Journal of Mathematics and Computer Science, 7(2013), 131-137.

[8] S. J. Wright, An infeasible interior point algorithm for linear complementary problems, Mathematical Programming, 62 (1996), 103-130.

[9] S. J. Wright, Primal--Dual Interior--Point Methods, SIAM, Philadelphia,1997. 